\title{
Los cambios que necesita EL SISTEMA DE ALTA DIRECCIÓN PÚBLICA
}

Por Eduardo Abarzúa* y Rafael Blanco**

Ph.D. en Ciencias del trabajo, Universidad Católica de Lovaina. Director Magister en y Negoocios UAH

creación del sistema de Alta Dirección Publica ha sido uno de los avances más del Estado de los últimos años. Esta institucionalidad ha permitido generar un proceso razonablemente transparente y merito"Maister on Derecho Público, PonIntegrante Consejo Alta Dirección Públita crático a la hora de seleccionar altos cargos en la administración del Estado. Gracias UAS nuevo sistema se ha creado un estamento écnico que convive con el actor politico y con el funcionario de carrera: el gerente publico profesional. Se trata de 942 cargos de ly II nivel jerarquico de servicios publicos adscritos a este medio (110 cargos de primer nivel jerarquico y 832 cargos de segundo nivel jerárquico). Asi, el Director

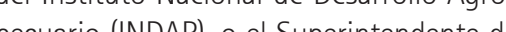
Salud, el Tesorero General de la Repic Salud, de SERVIU, son personas que han legas a sus cargos por un concurso público en el que intervienen empresas expertas en selección y búsqueda de personal, siendo los finalistas entrevistados por un Consejo politicamente plural que entrega una terna, cuaterna o quina al Presidente de la República, quien elige al titular de entre los candidatos que lograron pasar todas las etapas de evaluación.

El sistema, creado el 2003, ha mostrado su capacidad de atraer postulantes del se- 


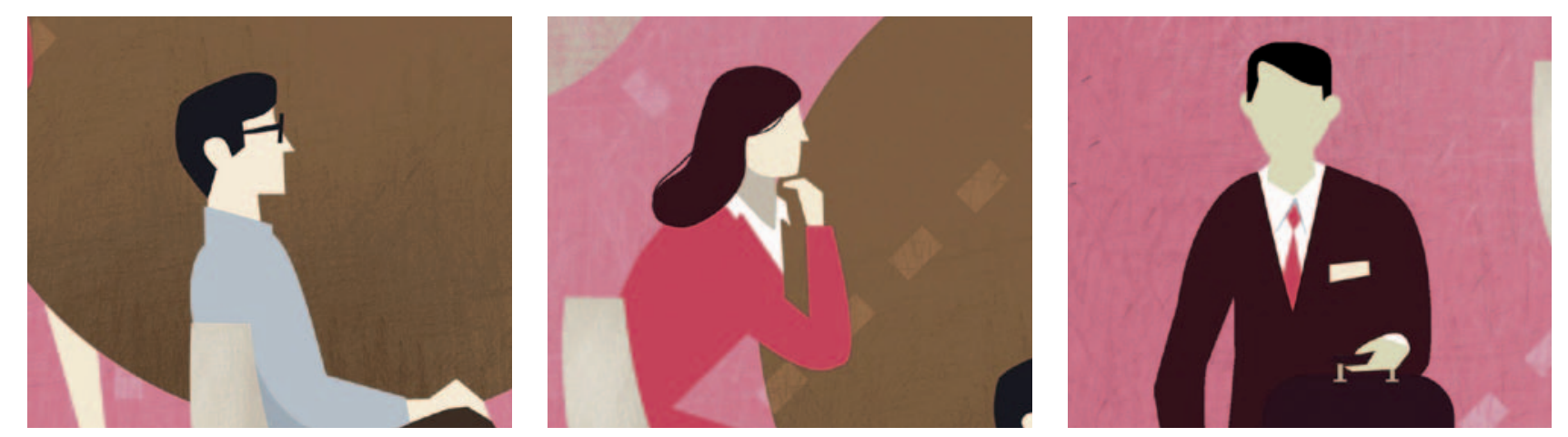

personas nombradas en cargos de l y II nivel de su nombramiento. jerárquico provenian del sector privado, en 2012 este porcentaje ascendio al $38 \%$ para mismo tiempo se ha creado una institucionalidad con prestigio y oficio en materias de selección de personal calificado para Estado, siendo reconocido el Consejo de Alta Dirección Pública (CADP) y la Dirección Nacional del Servicio Civil (DNSC).

En los últimos años el sistema ha estael gobierno entrante de Michelle Bacheles al cumplir un mes en el poder, haya despedido alrededor de $52 \%$ de los directivos públicos del I nivel jerárquico. Asimismo, si bien aún no existen datos oficiales, se sumarian despidos en el II nivel.

nuevo; el 2010 cuando asumió el Presidente Sebastián Piñera se desvinculó al $64 \%$ de los directivos de I nivel jerárquico en ejercicio y al 24\% del II nivel, cifras que siguieron creciendo en los años siquientes de gobierno. Lo anterior tiene enormes consecuencias presupuestarias, el 2010, se gastaro \$ 1.859 millones en costos asociados a ejecución de concursos pubblicos (publicaciones y consultoras) y en 2011 aumento este item en un $59 \%$, llegando a $\$ 2.952$ millones', presupuesto consistente con los cargos a cubrir luego de las desvinculaciones. Sin embargo, en los despidos antes descritos no hay falta alguna, se trata de de remoción de "exclusiva confianza y libre designacion" del Presidente de zepúblic o de la autoridad facultada despidos masivos

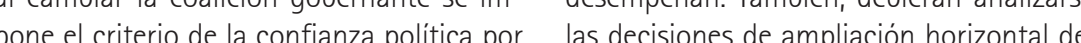
sistema ha instalado un estricto examen de incorporadas instituciones como el Comite idoneidad técnica del postulante al ingreso, de Inversiones Extranjeras, la Corporación enombramiento es una decisión política de Fomento de la Producción o la Superindel ejecutivo para aquellos que pasan dicho tendencia de Bancos e Instituciones Finanfiltro. Siendo asi, al cambiar la coalición go- cieras, entre otras. Además, y dado que se bernante, los profesionales nombrados en discute una descentralización efectiva, es a administración anterior dejarán de ser recomendable que la profesionalización gionales y municipios, identificando cargos que deben ser provistos via concurso púb co en el marco del SADP.

Respecto de los cargos de I nivel jerárquico que queden incluidos dentro del sistem Un podria contemplar sistemas de cuota. gos, quedaran excluidos de ser removidos en los cambios de gobierno. Por supuesto las excepciones estarian permitidas con acuerdo fundado del Consejo de ADP. Para los dos tercios de los cargos restantes, se debiera revisar alguna modalidad de concurso tipo fast track, en que el Consejo proponga una nómina a partir de su base de datos, con el fin de reducir los tiempos de conformación de nomina y los costos asociados al proceso, en los casos en que provista de urgencia

Respecto del II nivel jerárquico el foco debe estar en permear -con la lógica de prefesionalizacion y mento- las etapas confianza en el sistema y su capacidad de deben ser parte de la ADP y cuáles habría atraer a los mejores postulantes. En partiLeque sobre otras consideraciones Sibien nuestro sistema Recordemos que ain no han sido designación del Alto Directivo deba ser cular, resulta relevante revisar los aspectos Dirección Pública, quien debiera registrar la ligados a la evaluación del desempeño y la situación cuidando la confidencialidad.

tesvinculacion de los directivos, como los teria, en los úttimos años se han generado diversas propuestas que pueden ser parte de un proyecto de ley. En la práctica se trata de completar nuestro modelo de ADP hasta ahora de concursabilidad para cargos (position bosed), con criterios ligados a dar peso al desempeño y la gestión de los directivos Aparentemente, se ha logrado consenso en torno a la necesidad de traspasar la logica meritocratica que se aplica al momento de seleccionar, en particular, a la etapa de desvinculación. Es decir, equilibrar el despido fundado sólo en la perrdida de confianz politica, con argumentos basados en el desempenión de cume publico. El referente de

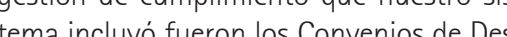
empeño, sin embargo, su uso ha sido prácticamente nulo. Es por ello que hace fal ta dotarlos de realidad institucional es decir protocolizar el proceso de discusión y definición de las metas de desempeño a cargo de los directivos, que vinculen los resultados institucionales y desempeño directivo. En este proceso, deben participar las autoridades gubernamentales y los directivos en de desempeno. Al mismo tiempo, se deberan contemplar procesos de seguimiento los convenios, de tal forma que sea posibe actualizar y corregir la formulación inicia plimiento esperado. Igualmente, el acto de despido debiera fundase en dicho referenla discusión y definición de los convenios desvins de suplentes -una vez ocurridas las minar el recurso de nombrar provisiones $y$ transitorios Lo anterior seria un auténtico desincentivo al uso de indiscriminado de la remoción por pérdida de confianza y también un estimulo a la pronta concursabilidad de los cargos. Por regla genera, estas plazas debieran ser cubiertas por subrogantes de planta mientras se realiza el concurso.

En materia de desvinculación, se debería aumentar la indemnización a 6 meses de remuneración cuando se pida la renuncia de manera anticipada, estableciendo as una autentica protección al directivo y un inpulso que ayuda a la postacion que a los funcionarios de planta del sector público, se les permita, en caso de ganar un concurso, guardar la titularidad de su cargo, a lo menos durante un periodo de ejercicio en el sistema de ADP.

Por supuesto existen otras medidas complementarias que van en la línea de lo ya expuesto. Si se materializa el desempeño como variable de retención o desvinculación de altos directivos públicos, es bastante probable que aumente la renovación de los periodos de ejercicio y algunos de ellos nuestro modelo no permite ninguna acción positiva para este grupo de disuna ación se have su salida de sistema. Par Io tanto ferencia de conocimientos. El paso de un pedelo de concurso a uno de desempeño, desde uno de empleo a uno de carrera. En mbos casos, son necesarias acciones difetes de gestión

Las propuestas anteriores implican un aumento del nivel de influencia y autonomia del Consejo de Alta Dirección Pública, de tal manera que sea un contrapeso efecvo en el fortalecimiento del sistema y ción. Además, un cambio en la ley puede ser una oportunidad para que la Dirección cional del Servicio Civil integre y concentre las diferentes funciones dispersas stado.

(n) te y ser validado frente al Consejo de A aseguren la retención del talento y la trans-
Balance de gestión integral año 2011. Dirección

Jorge Rodríguez Gross Fono Facultad: 28897366 e-mail: jrodrigu@
fen.uahurtado.cl 